University of Nebraska - Lincoln

DigitalCommons@University of Nebraska - Lincoln

Faculty Publications in Computer \& Electronics Electrical \& Computer Engineering, Department Engineering (to 2015)

\title{
Scalable Distributed Communication Architectures to Support Advanced Metering Infrastructure in Smart Grid
}

\author{
Jian Zhou \\ University of Nebraska-Lincoln, jzhou@unlserve.unl.edu \\ Rose Qingyang Hu \\ Utah State University, rose.hu@usu.edu \\ Yi Qian \\ University of Nebraska-Lincoln, yqian2@unl.edu
}

Follow this and additional works at: https://digitalcommons.unl.edu/computerelectronicfacpub

Part of the Computer Engineering Commons

Zhou, Jian; Qingyang Hu, Rose; and Qian, Yi, "Scalable Distributed Communication Architectures to Support Advanced Metering Infrastructure in Smart Grid" (2012). Faculty Publications in Computer \& Electronics Engineering (to 2015). 93.

https://digitalcommons.unl.edu/computerelectronicfacpub/93

This Article is brought to you for free and open access by the Electrical \& Computer Engineering, Department of at DigitalCommons@University of Nebraska - Lincoln. It has been accepted for inclusion in Faculty Publications in Computer \& Electronics Engineering (to 2015) by an authorized administrator of DigitalCommons@University of Nebraska - Lincoln. 


\title{
Scalable Distributed Communication Architectures to Support Advanced Metering Infrastructure in Smart Grid
}

\author{
Jiazhen Zhou, Member, IEEE, Rose Qingyang Hu, Senior Member, IEEE, and \\ Yi Qian, Senior Member, IEEE
}

\begin{abstract}
In this paper, we investigate the scalability of three communication architectures for advanced metering infrastructure (AMI) in smart grid. AMI in smart grid is a typical cyber-physical system (CPS) example, in which large amount of data from hundreds of thousands of smart meters are collected and processed through an AMI communication infrastructure. Scalability is one of the most important issues for the AMI deployment in smart grid. In this study, we introduce a new performance metric, accumulated bandwidthdistance product (ABDP), to represent the total communication resource usages. For each distributed communication architecture, we formulate an optimization problem and obtain the solutions for minimizing the total cost of the system that considers both the ABDP and the deployment cost of the meter data management system (MDMS). The simulation results indicate the significant benefits of the distributed communication architectures over the traditional centralized one. More importantly, we analyze the scalability of the total cost of the communication system (including MDMS) with regard to the traffic load on the smart meters for both the centralized and the distributed communication architectures. Through the closed form expressions obtained in our analysis, we demonstrate that the total cost for the centralized architecture scales linearly as $O(\lambda N)$, with $N$ being the number of smart meters, and $\lambda$ being the average traffic rate on a smart meter. In contrast, the total cost for the fully distributed communication architecture is $O\left(\lambda^{\frac{2}{3}} N^{\frac{2}{3}}\right)$, which is significantly lower.
\end{abstract}

Index Terms-Scalability, smart grid, advanced metering infrastructure (AMI), meter data management system (MDMS), facility location problem.

\section{INTRODUCTION}

YBER-PHYSICAL systems (CPSs) that utilize sensing, computation, communication, and control capabilities are widely being deployed in many application scenarios. Examples of the CPS systems include intelligent transportation systems, environment monitoring systems, and smart grid systems. A common feature of these systems is that a large amount of sensors are deployed over a wide area to implement the complex monitoring and control functions. Therefore, a main challenge is how to build a scalable communication architecture to handle the huge amount of data generated by those sensors.

As a typical example of a CPS, smart grid takes advantage of the advancement in communication and control technologies to create an automated, widely distributed delivery network through the use of bidirectional connection of electricity and information flows [1]. As the first major

- J. Zhou and Y. Qian are with the Department of Computer and Electronics Engineering, College of Engineering, University of Nebraska-Lincoln, Peter Kiewit Institute, 1110 South 67th Street, Omaha, NE 68182-0572. E-mail: \{jzhou10,yqian2\}@unl.edu.

- R.Q. Hu is with the Department of Electrical and Computer Engineering, Utah State University, 4120 Old Main Hill, Logan, UT 84321.

E-mail:rose.hu@usu.edu.

Manuscript received 23 Sept. 2011 ; revised 30 Dec. 2011; accepted 17 Jan. 2012; published online 27 Jan. 2012.

Recommended for acceptance by S. Papavassiliou, N. Kato, Y. Liu, C.-Z. $\mathrm{Xu}$, and $\mathrm{X}$. Wang.

For information on obtaining reprints of this article, please send e-mail to: tpds@computer.org, and reference IEEECS Log Number

TPDSSI-2011-09-0704.

Digital Object Identifier no. 10.1109/TPDS.2012.53. milestone and the fundamental step of the overall smart grid roadmap, an advanced metering infrastructure (AMI) is a system that measures, collects, and analyzes data about energy usage and power quality from the smart meters, and communicates with metering devices on request or on schedule [2]. AMI provides intelligent connections between consumers and system operators based on the information exchanged between the two sides. The vast amount of new data flowing from AMI allows improved management of utility assets, better planning of asset maintenance, additions and replacements, as well as better power quality and more reasonable price for customers. As an example, AMI can provide immediate feedback on predicting peak power usage and power quality, enabling the service provider to rapidly address grid demands.

Due to the pivotal role of information exchange in smart grid, data management is one of the most important tasks in smart grid. In AMI, there is a key component called meter data management system (MDMS). An MDMS is a database that performs long term data storage and management for the vast quantities of usage data and events [2]. It has analytical tools that enable the interaction with operation and management systems including outage management system (OMS), geographic information system (GIS), consumer information system (CIS) that manages the utility billing and customer information, and distribution management system (DMS) that provides power quality management and load forecasting based on meter data. A traditional AMI architecture is shown in Fig. 1, where a 


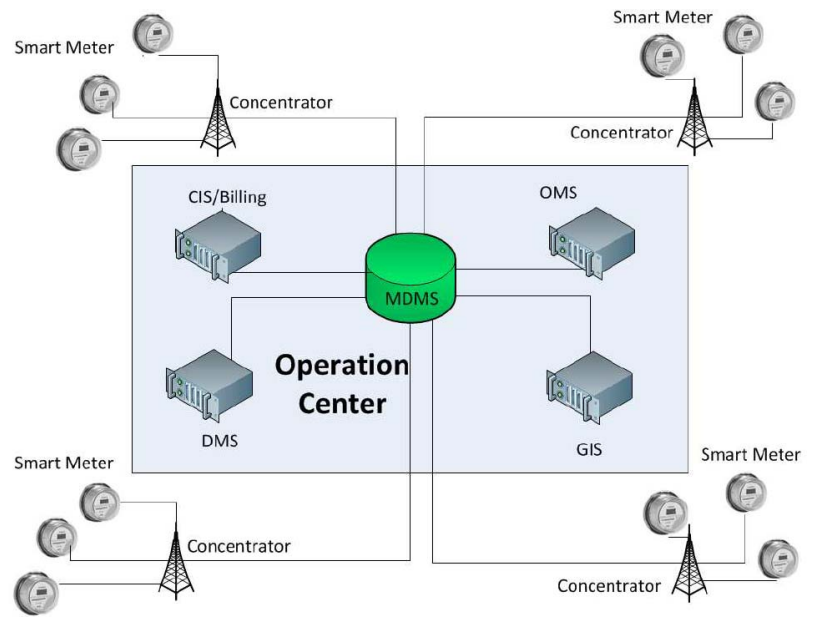

Fig. 1. A traditional AMI communication architecture in smart grid.

centralized MDMS is surrounded by the main operation and management services.

Our main focus in this paper is to investigate the communication cost of data collection and usage. Since MDMS is the entity to manage and process data in a smart grid system, our core task is to design a scalable architecture centered around MDMS by considering its interactions with smart meters and with the operation and management service gateways.

To study the scalability of a communication architecture in a smart grid system, we first define a new performance metric. Then, we formulate the new performance metric in terms of the communication architecture parameters, and investigate how the performance metric scales with the communication resources needed, which are mainly determined by the number of smart meters and the meter sampling frequency.

A good pervasive computing system design has to achieve scalability by severely reducing interactions between distant entities [3]. This is justified since long communication distance leads to a high communication resource usage and a high probability to encounter a bottleneck. Following the spirit of pursuing shorter communication distance for data migration, in this paper, we use a new performance metric to measure the scalability of an architecture for MDMS in smart grid: the accumulated bandwidth-distance product, or ABDP. The term bandwidth-distance product was used in the context of fiber-optic communication [4] (defined as the product of the length of a fiber-optic link and its maximum data rate) to help comparing the performance of different types of fiber-optic links. In a smart grid AMI system, however, we use the accumulated bandwidth-distance product to measure the total resources used to support communication demands.

The major contributions of this paper are in the following:

- We define a new performance metric for measuring the scalability of communication architectures in a smart grid AMI system.

- We propose an algorithm to minimize the total cost of an AMI communication system, which takes the deployment cost of MDMS into account.

- We analyze the scalability of three typical communication architectures for smart grid AMI systems. We demonstrate that the total cost for the centralized architecture scales linearly as $O(\lambda N)$, with $N$ being the number of smart meters, and $\lambda$ being the average traffic rate on a smart meter. In contrast, the total cost for the fully distributed communication architecture is just $O\left(\lambda^{\frac{2}{3}} N^{\frac{2}{3}}\right)$. Furthermore, the analysis matches very well with the simulation results obtained through the proposed algorithm.

The rest of this paper is organized as follows: in Section 2, the background and related work regarding the scalability of communication infrastructures in smart grid AMI is presented. In Section 3, two distributed communication architectures for the smart grid systems are presented. Optimization problems to minimize the total cost of the system are formulated, and the solution algorithms are proposed in Section 4. In Section 5, the scalability of the distributed and the traditional centralized communication architectures are analyzed and asymptotic closed form expressions for the ABDP and total costs are derived for both architectures. The performance evaluation in Section 6 verifies the accuracy of the asymptotic analysis and demonstrates the benefits of the distributed communication architectures in terms of lower cost and better scalability. Section 7 concludes this paper.

\section{Background and Related Work}

Scalability is the ability of a system to handle growing amounts of work in a graceful manner [5], [6]. It can be measured in various dimensions such as administrative scalability, functional scalability, geographic scalability, and load scalability [5]. In this paper, we mainly concern the load scalability, i.e., the easiness for a system to increase its resources to accommodate the increasing load.

It is necessary to define the specific requirements for scalability on those dimensions in different systems. For instance, in the area of Internet routing, one measurement of scalability of a routing algorithm is how the routing table on a router scales with the number of nodes (customers) of the whole Internet.

Since scalability also means that the system must be economically deployable in a wide range of sizes and configurations [7], we propose to use ABDP to evaluate the scalability of a communication architecture in a smart grid AMI system. As we stated in the previous section, the ABDP is a measurement of communication resources needed for supporting certain communication needs. It integrates two important metrics, the communication bandwidth and the communication distance. For the communication architectures studied, we will evaluate how the resulted ABDP scales with the number of smart meters and the sampling frequency on smart meters.

\subsection{Why We Need a Scalable Communication Architecture}

In many of the smart grid AMI systems that have been deployed, data is collected from smart meters every 15 minutes [8]. Although this is a significant improvement compared with the traditional way that only records the meter data once a month, it is far from enough in achieving the full vision of a smart grid, especially for the goals like advanced distribution automation (ADA) and advanced asset management (AAM) [2]. As discussed in [9], to support the voltage and voltage adjust rheostat (VAR) 
control service in ADA, voltage and current root mean square (RMS) values measured at the substation and voltage RMS values measured at the end of the feeder should be collected every 30 seconds. Data available from regular meters of the customers, which is the averaged RMS value over a 15-minute interval, is not sensitive enough for this application [9]. As another example, for the outage management application, ideally we should be able to obtain real time data from smart meters to prevent cascading outage [10] and to recover from outage in the shortest time.

In summary, with the overall modern smart grid roadmap being implemented step by step, both the number of smart meters and the sampling frequency on a meter will increase dramatically. Consequently, huge amount of data traffic will go through the MDMS and post a great challenge on the scalability of the traditional smart grid AMI communication architecture.

\subsection{Literature on Scalable Architectures in Smart Grid}

There are different focuses on the scalability issues in smart grid. In [11], Arenas-Martinez et al. investigated the scalability problem from the aspects of data storage and monthly bill processing. They mainly considered the scalability of the processing ability with the number of meters. The main storage techniques they compared are the centralized relational database, the distributed relational database, and the file systems. The sampling interval at a smart meter was assumed to be 15 minutes. Since the main focus was on the scalability of processing ability, the model was highly abstractive. For example, the concentrator node they defined is different from the standard definition in [2]. It is more like the MDMS instead of the concentrator that acts as the access point for smart meters. Moreover, they only considered the logical locations of databases (i.e., located at the concentrator or in the operation center). The physical locations of databases and distances between different locations were not considered. In contrast, our main concern is how the communication cost (in terms of the ABDP value) scales with the number of smart meters and the sampling frequency. The physical location and distance are the vitally important factors to be considered in designing a scalable communication architecture.

In [12] and [13], the authors proposed to employ the computing service to support smart grid applications and discussed the main challenges. A major advantage of cloud computing service is its flexibility in providing powerful storage, processing, and communication ability for the future smart grid applications. In that sense, cloud computing can offer a scalable platform for smart grid systems. While the main focus of the cloud computing approaches [12], [13] is on providing adequate resources to support smart grid applications, they did not study the optimal deployment of the necessary resources. For the scalable architectures that we are investigating in this paper, we emphasize on the design of optimal communication infrastructures for smart grid AMI.

\subsection{Scalable Communication Architectures in Internet}

The client-server architecture has been dominant for providing services in Internet, for example, web services and file sharing services. This was not a problem at the earlier stage (e.g., in early 1990s) since the number of Internet users was limited. However, with the explosive increase of Internet applications and users since late 1990s, this traditional centralized architecture would no longer be a good choice as it does not scale well. For example, to provide web services in Internet to a large number of users widely distributed, a centralized server (even server farms) will have serious communication bottlenecks mainly due to the long paths traversed by the information flows as well as high-level aggregation of information flows when they get close to the server. Furthermore, as it will be demonstrated for smart grid AMI systems in this paper, the centralized communication architecture also has a much higher bandwidth waste compared with the distributed communication architectures to be presented in Section 3.

To overcome the nonscalable problem of the client-server architecture, two popular distributed communication architectures emerged for different applications in Internet, namely, content distribution networks (CDNs) [14] and peer-to-peer networks (P2P) [15]. CDNs are used to offer services to business partners, mainly for boosting the performance of web applications used by a large number of users all over the world. They are usually well planned and deployed, and the distributed servers are limited in numbers but powerful in each single server. In contrast, P2P services are autonomous, in which spontaneous nodes can be sources of contents. In other words, each node can act as either a client or a server or both. With multiple data sources exploited, desirable performance can be achieved at each user node although the processing ability and communication bandwidth at each node are limited. Due to the need to exploit a huge number of nodes to provide an ideal service, the process to locate the source of contents could be very costly and an inappropriate design may cause serious scalability problems [16], [17], [18].

A key problem in the design of a CDN is the placement of web server replicas. In [19], the optimal placement of a given number of web server replicas in a CDN is studied. It is modeled as a K-median problem, and various placement algorithms, including a greedy algorithm, a hot spot algorithm, and a tree-based algorithm, are evaluated by simulating their behaviors on synthetic and real network topologies. In addition, the sensitivity of the placement algorithms to imperfect knowledge of client workload characteristics is studied. It is concluded that a greedy algorithm for solving the web server replica placement problem can provide a CDN with performance that is close to optimal. In another related work [20], Jamin et al. study the optimal placement of tracers that are used to measure and build a distance map in Internet. They compare different heuristics when there is no knowledge about the Internet topology, including placing the tracers on the stub Autonomous Systems, or on the transit Autonomous Systems, or on both.

The communication architectures that we are proposing for smart grid systems have some similarities with the CDNs. Both require a small number yet powerful servers to be deployed. However, their differences are also prominent as discussed in the following:

- Information collection and usage mode. In CDNs, contents are assumed to be transferred from a central server at a low frequency, and thus can often 
be neglected compared with the traffic incurred by information usage. In contrast, in smart grid large amount of data are collected at a higher frequency from the smart meters that are widely distributed, while the amount of information needed by the operation center varies depending on the specific applications.

- Measurement of scalability. In CDNs, the main concern is how to maintain high performance of user's access to the web applications. Actually, the scalability issue was not explicitly studied in the literature of CDNs, mainly because it is difficult to define the scalability in terms of the access performance metric such as delay. While for smart grid in this paper, we mainly concern the scalability in terms of the communication resources used, such as the number of smart meters and the meter data sampling frequency.

A major difference in our work compared with previous work is that we not only study the optimal placement of distributed MDMS in smart grid systems, but also give a theoretical analysis on how the distributed and the centralized communication architectures scale with different load parameters, e.g., the number of smart meters and the sampling frequency on each meter.

In summary, scalability is an important issue that needs to be addressed in smart grid communication infrastructures. To the best of our knowledge, although there have been previous studies in the related areas like the scalability of storage and processing abilities in smart grid and the scalable communication architectures in Internet, they did not directly address the communication infrastructure scalability issue in smart grid.

\section{Distributed Communication Architectures FOR SMART GRID}

As we discussed in the previous section, scalability of a communication architecture in smart grid is a very important but not well-addressed issue. In this paper, we investigate and analyze the scalability of several different smart grid AMI architectures.

In a centralized architecture as illustrated in Fig. 1, the MDMS is located in the operation center. This facilitates the management of equipments and data, and thus all the operation and management services can fetch data from a single server in the fastest way. However, since data collected from the smart meters needs to go through the centralized MDMS, it makes the system nonscalable in several dimensions. First, it is nonscalable in terms of performance of data communications. It is highly possible to have bottlenecks in the zones that are close to the operation center. A large communication delay and even a significant loss of data may occur when congestion happens. Second, it is nonscalable in terms of data processing. As pointed out by Gerdes et al. [23], due to the large data load, centralized processing becomes less and less feasible. Third, it is nonscalable in terms of communication resources used. Since almost each message needs to go through a long path before arriving at the MDMS, it causes a huge waste of communication resources. As an alternative, a distributed architecture may significantly reduce the needed communication bandwidth as well as the communication distance.

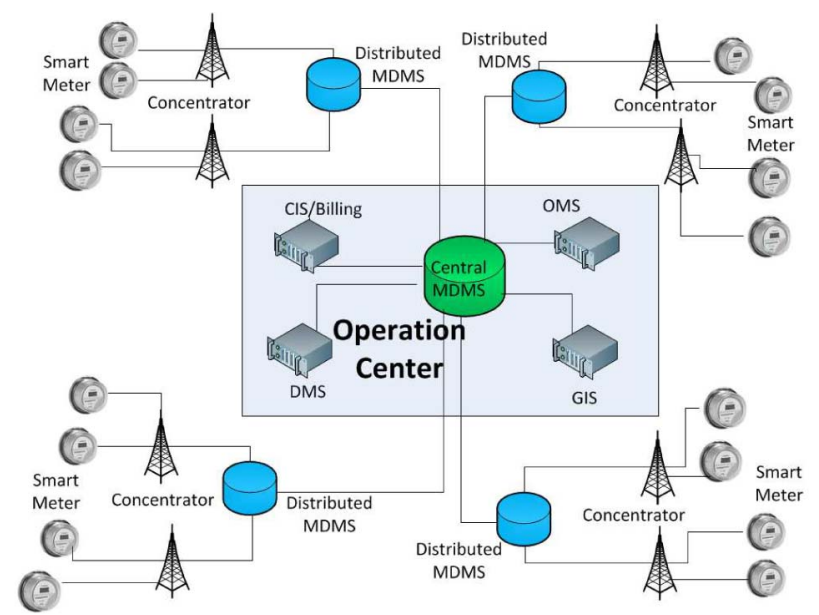

Fig. 2. A communication architecture with distributed MDMS in smart grid.

Our focus in this paper is on the scalability in terms of communication resources needed. In this section, we present two distributed communication architectures that can improve the scalability in that aspect. In the first architecture, multiple distributed MDMS are deployed in the entire smart grid AMI service area under study. In the second architecture, both MDMS and operation and management servers are decentralized.

\subsection{A Communication Architecture with Distributed MDMS}

As shown in Fig. 2, multiple distributed MDMSs are deployed close to smart meters, with each MDMS being responsible for storing and processing data from the smart meters in the specific surrounding area through multiple concentrators. The communication distance for information collection is largely shortened compared with the centralized architecture, and the corresponding resources needed (measured by the ABDP) are reduced significantly. On the other hand, although the communication distance for information usage increases, the raw information has been processed and consolidated at the distributed MDMSs. Thus, the communication bandwidth needed for information usage is reduced compared with that for the raw data in a centralized architecture. This leads to a possibly significant saving of total communication resources.

The data needed by each type of service in the operation center is the refined data that has been processed by each MDMS, which is significantly lower than the raw data collected from the large number of smart meters. Let $\alpha$ be the ratio of the refined data needed by operation and management services compared with the raw data collected by the distributed MDMS. Usually $\alpha$ is a small value since only a small portion of the data is needed by the operation and management services. For instance, the outage management service (OMS) only needs to obtain data when the power quality (measured in current and voltage) at a customer site is abnormal. So most data, especially those that have been collected at a high frequency, are not needed at the OMS and will be filtered out.

\subsection{A Fully Distributed Communication Architecture}

The communication cost in the architecture shown in Fig. 2 could be further reduced if the original operation and 


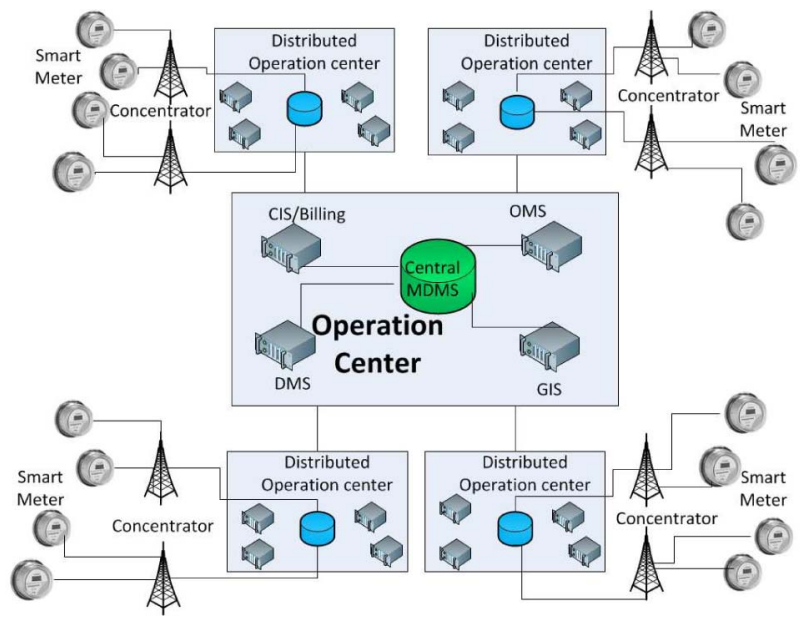

Fig. 3. A fully distributed communication architecture in smart grid.

management server is also decentralized. As shown in Fig. 3, a practical deployment in this fully distributed communication architecture is to colocate one operation and management server with each MDMS.

In the fully distributed communication architecture described above, the communication cost between a distributed operation and management server and its associated MDMS can be neglected since they are physically located together. The communication bandwidth needed is mainly for the information exchange between each distributed server and the central server, which can be viewed as a constant since it will not be affected by the number of smart meters or the sampling frequency on a smart meter. For instance, if there is an outage management server at each of the distributed MDMS, most abnormal situations can be handled locally. The only information that needs to be reported to the centralized OMS server is the summary information about customer's power quality in the area managed by this MDMS. This summary information does not change with the number of users or the sampling frequency. In other words, the data that needs to be transferred from a distributed OMS to the centralized OMS can be viewed as a constant.

In the distributed communication architectures presented in Figs. 2 and 3, the optimal number and locations of distributed MDMS to be deployed need to be determined. We will formulate two optimization problems, one for each architecture, in Section 4. The heuristic solutions will be provided to the optimization problems in the same section. The scalability of the distributed communication architectures will be analyzed in Section 5 .

\section{Optimal Deployment of Distributed MDMS}

In this section, two optimization problems are formulated to find the optimal deployment of the distributed MDMSs. A heuristic algorithm is then provided to obtain the close-tooptimal solutions.

\subsection{Assumptions and Problem Formulation for the Distributed MDMS Architecture}

The key parameters and variables used in the optimal formulation are denoted as following: $\lambda_{i} \quad$ Message generation rate on each concentrator $i$ in the unit of megabits/second (Mbps).

$d_{i j} \quad$ Distance from concentrator $i$ to MDMS $j$ in the unit of kilometer (Km).

$C_{j} \quad$ Distance from MDMS $j$ to the operation center.

$x_{i j}$ Indicator: whether concentrator $i$ is connected to MDMS $j$.

$A \quad$ The size of a $a \times a$ square area, i.e., $A=a^{2}$.

$\mathrm{N}$ Total number of concentrators.

M Total number of candidate locations for MDMS.

$\alpha \quad$ The ratio of refined data needed by operation and management services over the raw data collected by MDMS.

$\beta \quad$ Unit cost of bandwidth-distance product $(M b p s \times K m / \$)$.

$\gamma \quad$ Bandwidth needed for information exchanged between a distributed server and a centralized server.

$y_{j} \quad$ Whether candidate location $j$ is chosen to deploy a MDMS.

$F_{j} \quad$ Cost of deploying a MDMS at location $j$.

In the distributed communication architecture shown in Fig. 2, the ABDP of a data source mainly includes two parts, 1) information collection ABDP, which counts the cost from each concentrator to the corresponding distributed MDMS, and 2) information usage ABDP, the communication cost from each MDMS to the operation center. The local communication cost from the smart meters to the concentrators is neglected since the corresponding distance is very small compared with the distance between a concentrator and an MDMS. Similarly, the communication cost between the centralized MDMS and operation and management servers is also neglected.

For a concentrator $i$ that is connected through MDMS $j$, the information collection ABDP can be expressed as $\lambda_{i} d_{i j}$, and the corresponding information usage ABDP is $\alpha \lambda_{i} C_{j}$. When we consider the total ABDP contributed by all the $N$ concentrators, the first part is $\sum_{j=1}^{M} \sum_{i=1}^{N} \lambda_{i} d_{i j} x_{i j}$, while the second part is $\sum_{j=1}^{M} \alpha \sum_{i=1}^{N} \lambda_{i} x_{i j} C_{j}$. The variable $x_{i j}$ is applied since $\lambda_{i} d_{i j}$ and $\alpha \lambda_{i} C_{j}$ will be counted only when concentrator $i$ is connected through MDMS $j$.

We assume that the ABDP is proportional to the deployment cost of the corresponding equipments (e.g., optical fibers and routers), the cost can be obtained by multiplying the ABDP by a unit cost $\beta$. Thus, the objective function of this optimization problem is obtained by adding the deployment cost of the distributed MDMS with the cost of needed ABDP.

As a result, the optimization problem to minimize the total cost of the system can be formulated as

$$
\operatorname{Min} \beta\left(\sum_{j=1}^{M} \sum_{i=1}^{N} \lambda_{i} d_{i j} x_{i j}+\sum_{j=1}^{M} \alpha \sum_{i=1}^{N} \lambda_{i} x_{i j} C_{j}\right)+\sum_{j=1}^{M} F_{j} y_{j},
$$

s.t.

$$
\sum_{j=1}^{M} x_{i j}=1
$$




$$
\begin{aligned}
& x_{i j}=0 \text { or } 1, \\
& y_{j}=0 \text { or } 1 .
\end{aligned}
$$

Note that in the formulation above we assume there is no capacity constraint with each MDMS. This is reasonable at the planning stage since our goal is to minimize the total cost.

The optimization solutions will provide the candidate MDMS locations that are eventually selected. MDMS $j$ is chosen if $y_{j}=1$. The constraint conditions (2) and (3) enforce that a concentrator can be only connected to a single MDMS, so that data collected from the same meter or the meters from the same area is stored together and can be fetched from the same MDMS. This design will guarantee desirable performance of the operation and management services such as outage management since we may often need to collect abnormal information from the smart meters in a large but connected area.

\subsection{A Greedy Algorithm}

The optimization formulation in (1)-(4) can be viewed as a variation of the uncapacitated facility location problem that tries to find the optimal number and locations of warehouses to minimize the total cost [21]. Since the facility location problem is NP-hard, there is no effective method to obtain exact optimal solution for a large size problem [22]. As a practical solution, heuristic methods or approximate algorithms (for instance, a greedy algorithm and a local search method) are widely employed. A well-known example of using a greedy algorithm for the facility location problem is presented in [21]. As another example, in [19], Qiu et al. formulated the web cache replica placement problem into a K-median problem and applied a greedy algorithm. Note that K-median problem is a variation of the facility location problem that focuses on finding the optimal locations of the facilities by assuming the number of facilities is given.

In the following, we present a greedy algorithm in Algorithm 1 to find the close-to-optimal solutions for the MDMS location problem. It is an extension to the greedy algorithm in [19] and [21] by considering the specific objective function and constraints shown in (1)-(4).
Algorithm 1. GREEDY ALGORITHM
Input: (1) Candidate locations for distributed MDMS
(2) Deployment cost of each MDMS
Output:
(1) Minimum total cost
(2) Deployment of distributed MDMS

1: $m=0 / /$ The number of distributed MDMS selected

2: while $m<M$ do

3: $\quad m=m+1$

4: $\quad$ Find a new candidate location that minimizes (1) while satisfying $\sum_{j=1}^{M} y_{j}=m$

5: $\quad$ For each concentrator $i$, assign it to MDMS $j$ (out of the $m$ ones already chosen) such that $\lambda_{i} d_{i j}+\alpha \lambda_{i} C_{j}$ is minimized

6: Compute the total cost under the current assignment

7: $\quad$ Record the new lowest cost value if it is lower than 8: end while
9: Output the minimal total cost and the selected locations for MDMS

In Algorithm 1, we find the optimal locations of MDMSs for each given value $m$, with $m$ from 1 to $M$. At the first step, all $M$ possible locations are tested and the one that minimizes the objective cost function in (1) is selected as the location for the first MDMS. In the $m$ th step, an optimal location for a new MDMS is found in addition to those already found in the previous steps to minimize the total cost. When considering each new candidate location, each concentrator $i$ is assigned to one of the $m$ MDMSs (the $m-$ 1 ones already selected and the one under consideration) so that $\lambda_{i} d_{i j}+\alpha \lambda_{i} C_{j}$ is minimal. This process will be repeated until that all $M$ locations are tested. The number and locations of MDMSs that lead to the minimum cost will be the solution.

Compared with the brute force optimization method that checks every possibility to get the exact optimization result, the greedy algorithm is much simpler as it selects only one new location in each step. This makes sure that the complexity of the algorithm grows polynomially with the number of candidate locations $M$. In contrast, the brute force optimization method will select $m$ new locations in step $m$. This means that $\left(\begin{array}{l}M \\ m\end{array}\right)$ combinations of possible locations will be checked in step $m$. As a result, the computing complexity will grow exponentially in terms of $M$ and is much less scalable for a large size problem.

It is also worthy to mention that, although the algorithm presented above needs the full knowledge about the size of the smart grid AMI area, the locations of concentrators, and the sampling frequency on each smart meter, all these information is normally accessible at the operation center.

\subsection{The Fully Distributed Communication Architecture}

For the fully distributed communication architecture, the information usage cost is $\sum_{j=1}^{M} \gamma C_{j} y_{j}$. As a result, the objective function is

$$
\operatorname{Min} \beta\left(\sum_{j=1}^{M} \sum_{i=1}^{N} \lambda_{i} d_{i j} x_{i j}+\sum_{j=1}^{M} \gamma C_{j} y_{j}\right)+\sum_{j=1}^{M} F_{j} y_{j}
$$

The constraints are the same as in (2)-(4).

The algorithm for solving this optimization problem is similar to Algorithm 1. The only difference is in Step 5, where the values of $\lambda_{i} d_{i j}$ are compared when determining which MDMS should a concentrator be assigned to.

\section{SCALABILITy ANALYSIS}

The greedy algorithm introduced in the previous section provides a practical optimization method in solving practical infrastructure deployment problems. In this section, we further analyze the scalability of the centralized and the two distributed communication architectures. Note that the analysis is asymptotic, and we assume that there is no up limit on the number of distributed MDMSs.

\subsection{Centralized Communication Architecture}

When the centralized communication architecture is employed, only one MDMS is used. Since the information 
usage cost in this case can be omitted due to the short communication distance between the MDMS and the operation and management server, the total cost shown in (1) is reduced to

$$
\beta \sum_{i=1}^{N} \lambda_{i} D_{i}+F_{0}
$$

In the above expression, $D_{i}$ is the distance between concentrator $i$ and the central MDMS, and $F_{0}$ represents the deployment cost for the central MDMS. In practice, the distance between an MDMS and a concentrator or operation center is the length of the path that goes through the route. However, for analysis, we assume that it is the direct distance between the two locations.

The main computation part in (6) is to compute $\sum_{i=1}^{N} \lambda_{i} D_{i}$. Since both $\lambda_{i}$ and $D_{i}$ can be viewed as random variables, according to the definition of the expected value (denoted as $E[$ ) for discrete random variables, we have

$$
E\left[\lambda_{i} D_{i}\right]=\lim _{N \rightarrow \infty} \frac{\sum_{i=1}^{N} \lambda_{i} D_{i}}{N} .
$$

On the other hand

$$
E\left[\lambda_{i} D_{i}\right]=E\left[\lambda_{i}\right] E\left[D_{i}\right]=\overline{\lambda D} .
$$

Here, $\bar{\lambda}$ is the average traffic rate on concentrators and $\bar{D}$ is the average distance between a concentrator and the central MDMS.

With a large $N$, we can derive the following expression on the ABDP for the centralized architecture (denoted as $\left.A B D P_{C}\right)$ based on (7) and (8)

$$
A B D P_{C}=\sum_{i=1}^{N} \lambda_{i} D_{i} \approx \bar{\lambda} D N .
$$

As a result, the total cost in the traditional centralized communication architecture can be approximated as

$$
\text { TotCost } \approx \beta \bar{\lambda} \bar{D} N+F_{0} .
$$

This means that under the centralized communication architecture, both the total cost and the ABDP value scale linearly with all of the following three variables: the number of concentrators (also the number of smart meters), the average data generation rate on a concentrator, and the average distance between a concentrator and the central MDMS.

\subsection{Communication Architecture with Distributed MDMS}

With the distributed MDMS architecture employed, we denote the optimal number of MDMS as $M_{o}$ that minimizes the total cost. As an asymptotic analysis, we assume that the area covered by a smart grid AMI system is correspondingly divided into $M_{o}$ subregions with equal sizes. The number of concentrators in each subregion is $\frac{N}{M_{o}}$, and the size of each subregion is $\frac{A}{M_{0}}$.

In each subregion $j$ that has $\frac{N}{M_{o}}$ concentrators, the ABDP value for information collection can be written as

$$
\sum_{k=1}^{\frac{N}{M_{o}}} \lambda_{k} d_{k j}
$$

Denote $\overline{d_{j}}$ as the average distance between a concentrator in subregion $j$ and MDMS $j$, and assume that the average traffic rate in each subregion is the same as that of the whole area. Similar to the derivation for the centralized communication architecture, the ABDP value of a subregion $j$ can be approximated as

$$
\sum_{k=1}^{\frac{N}{M_{o}}} \lambda_{k} d_{k j} \approx E\left[\lambda_{k} d_{k j}\right] \frac{N}{M_{o}}=\overline{\lambda d_{j}} \frac{N}{M_{o}} .
$$

The ratio of the average distance between a concentrator in subregion $j$ and its corresponding MDMS over the length of a side in subregion $j$ should be the same as the ratio for the whole area. Since the size of a subregion is $\frac{A}{M_{o}}$, the length of each side (assume that it is also a square area) is $\sqrt{\frac{A}{M_{o}}}=\frac{a}{\sqrt{M_{o}}}$. Thus,

$$
\overline{d_{j}}=\frac{\bar{D}}{a} \frac{a}{\sqrt{M_{o}}}=\frac{\bar{D}}{\sqrt{M_{o}}} .
$$

Substitute (13) into (12), we have

$$
\sum_{k=1}^{\frac{N}{M_{o}}} \lambda_{k} d_{k j} \approx \frac{\bar{\lambda} \bar{D} N}{M_{o}^{\frac{3}{2}}} .
$$

In a similar way, the ABDP for information usage cost in (1) can be estimated as

$$
\alpha \sum_{j=1}^{M} \sum_{i=1}^{N} \lambda_{i} x_{i j} C_{j} \approx \alpha \frac{N}{M_{o}} \bar{\lambda} \sum_{j=1}^{M_{o}} C_{j} .
$$

If we define $\bar{C}$ as the average distance between a distributed MDMS and the operation center, we can approximate it as $\bar{D}$, which is the average distance between a concentrator and the operation center, then the above expression becomes

$$
\alpha \sum_{j=1}^{M} \sum_{i=1}^{N} \lambda_{i} x_{i j} C_{j} \approx \alpha \overline{\lambda D} N .
$$

Substitute (14) and (16) into (1), we have

$$
\begin{aligned}
\text { TotCost }= & \sum_{j=1}^{M_{o}} \frac{\beta \overline{\lambda D} N}{M_{o}^{\frac{3}{2}}}+\alpha \beta \bar{\lambda} \bar{D} N+\sum_{j=1}^{M_{o}} F_{j} \\
& \approx \frac{\beta \overline{\lambda D} N}{M_{o}^{\frac{1}{2}}}+\alpha \beta \overline{\lambda \bar{D}} N+M_{o} \bar{F} .
\end{aligned}
$$

Note that $\bar{F}$ is the average deployment cost of a distributed MDMS. To guarantee the value of (17) is minimum, $M_{o}$ should be

$$
M_{o}=\left(\frac{\beta \bar{\lambda} \bar{D} N}{\bar{F}}\right)^{\frac{2}{3}}
$$

As a result, the optimal total cost can be approximated by

$$
\text { TotCost }=2(\beta \overline{\lambda D} N)^{\frac{2}{3}} \bar{F}^{\frac{1}{3}}+\alpha \beta \overline{\lambda D} N .
$$

If we consider the ABDP in the distributed communication architecture (denoted as $A B D P_{D}$ ), it can be approximated as 


$$
\begin{aligned}
A B D P_{D} & =\sum_{j=1}^{M}\left(\sum_{i=1}^{N} \lambda_{i} d_{i j} x_{i j}+\alpha \sum_{i=1}^{N} \lambda_{i} x_{i j} C_{j}\right) \\
& \approx \frac{\overline{\lambda \bar{D} N}}{M_{O}^{\frac{1}{2}}}+\alpha \bar{\lambda} \bar{D} N \\
& =(\overline{\lambda \bar{D}} N)^{\frac{2}{3}}\left(\overline{\bar{F}} \frac{\bar{\beta}}{{ }^{\frac{1}{3}}}+\alpha \overline{\lambda \bar{D}} N .\right.
\end{aligned}
$$

It can be observed that, both the ABDP and the total cost for information collection under a distributed MDMS architecture scales at $x^{\frac{2}{3}}$ in terms of the number of concentrators $N$, the average traffic rate on each concentrator $\bar{\lambda}$, and the average distance between a concentrator and the central MDMS, which is proportional to the single side length of the area. In contrast, the information usage cost, which is the second part in (19) and (20), scales linearly in terms of the three parameters. When $\alpha$ is small, the distributed MDMS communication architecture has much better scalability compared with the centralized one. In fact, when $N \rightarrow \infty$, the first term in (19) and (20) can be neglected compared with the second term. This means that both the total cost and the ABDP value of the distributed architecture is $\alpha$ times of that of the centralized architecture.

\subsection{The Fully Distributed Communication Architecture}

Similar to the analysis for the distributed MDMS architecture as presented above, under an optimal design that has $M_{o}$ distributed operation centers, the total cost computed in (5) can be estimated as

$$
\begin{aligned}
\text { TotCost } & =\sum_{j=1}^{M_{o}}\left(\frac{\beta \overline{\lambda D} N}{M_{o}^{\frac{3}{2}}}+\gamma \beta C_{j}\right)+\sum_{j=1}^{M_{o}} F_{j} \\
& \approx \frac{\beta \overline{\lambda D} N}{M_{o}^{\frac{1}{2}}}+\gamma \beta M_{o} \bar{D}+M_{o} \bar{F} .
\end{aligned}
$$

To minimize the total cost, $M_{o}$ should be

$$
M_{o}=\left(\frac{\beta \overline{\lambda D} N}{\gamma \beta \bar{D}+\bar{F}}\right)^{\frac{2}{3}} .
$$

As a result, the optimal total cost can be approximated by

$$
\text { TotCost }=2(\beta \bar{\lambda} \bar{D} N)^{\frac{2}{3}}(\gamma \beta \bar{D}+\bar{F})^{\frac{1}{3}} .
$$

Correspondingly, the ABDP value of this architecture is

$$
A B D P_{F} \approx(\overline{\lambda \bar{D}} N)^{\frac{2}{3}}\left(\gamma \bar{D}\left(\frac{\beta}{\gamma \beta \bar{D}+\bar{F}}\right)^{\frac{2}{3}}+\left(\frac{\gamma \beta \bar{D}+\bar{F}}{\beta}\right)^{\frac{1}{3}}\right) .
$$

It can be observed that the total cost and the ABDP of this fully distributed architecture will scale at $x^{\frac{2}{3}}$ in terms of the number of concentrators $N$, and the average traffic rate on each concentrator $\bar{\lambda}$. This means that the fully distributed architecture is more scalable than the distributed MDMS architecture.

\section{Performance Evaluations}

\subsection{Simulation Setup}

We consider a basic evaluation scenario in which 2,500 concentrators are uniformly distributed in a $100 \times 100 \mathrm{Km}$

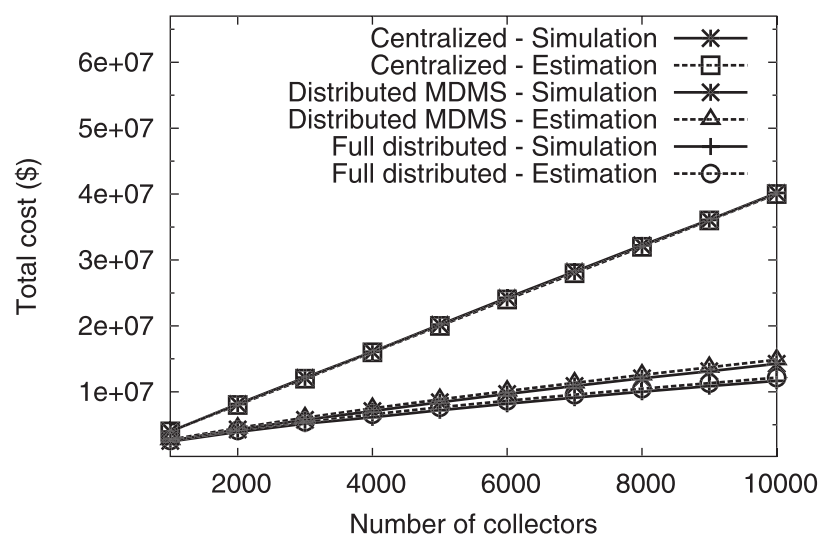

Fig. 4. Comparison of simulation and theoretical estimation results for the total cost.

area. There are 100 candidate locations for the distributed MDMS, which are evenly spaced in the same area. The operation center is located at the center.

This scenario corresponds to a city with about 1 million population, with the assumption that each family (average size is 4 persons / family) owns a smart meter. The number of smart meters covered by each concentrator is 100 on average.

As a widely used source for the volume of data generated by a smart meter, currently about 400 Mega bytes are generated a year per meter and data is collected every 15 minutes [8]. So roughly 12K Bytes of data are collected every time. Since we aim at future applications, the sampling frequency needs to be largely increased to avoid disastrous events such as cascading outage. Thus, we assume that the sampling period at each meter is 10 seconds, which corresponds to a data rate of about 10 kilobits / second (Kbps) per meter. As a result, the average data rate at a concentrator is $1 \mathrm{Mbps}$.

\subsection{Performance Results}

In Section 5, the scalability of the centralized and the two distributed communication architectures is analyzed and estimated. In the following, we verify the accuracy of the estimation.

We conduct experiments for different $N$ values, from 1,000 to 10,000 . The deployment cost of each MDMS is assumed to be $\$ 100,000$, and $\alpha=0.1$. The unit cost for the accumulated bandwidth-distance product is assumed to be $\beta=\$ 100$ (per Mbps $\times \mathrm{Km}$ ). The bandwidth needed between each distributed operation center and the central one is $\gamma=10 \mathrm{Mbps}$. Note that the average distance between a concentrator and the central MDMS is estimated as $\bar{D} \approx$ $0.4 a$ in the computation since the area we considered is a square (it is $\frac{2 r}{3}$ if the area is a circle with radius $r$ ). As shown in Fig. 4, the total costs computed through (10), (19), and (23) are compared with the simulation results by running Algorithm 1 and the algorithm for the fully distributed architecture. It can be seen that the theoretical estimations match the simulation results very well for a wide range of $N$ values, which confirms the validity of our asymptotic theoretical analysis. Furthermore, the comparison on ABDP value is presented in Fig. 5 and it also matches the theoretical estimations well.

In the following, we conduct more simulations based on Algorithm 1 to show how the total cost of the distributed 


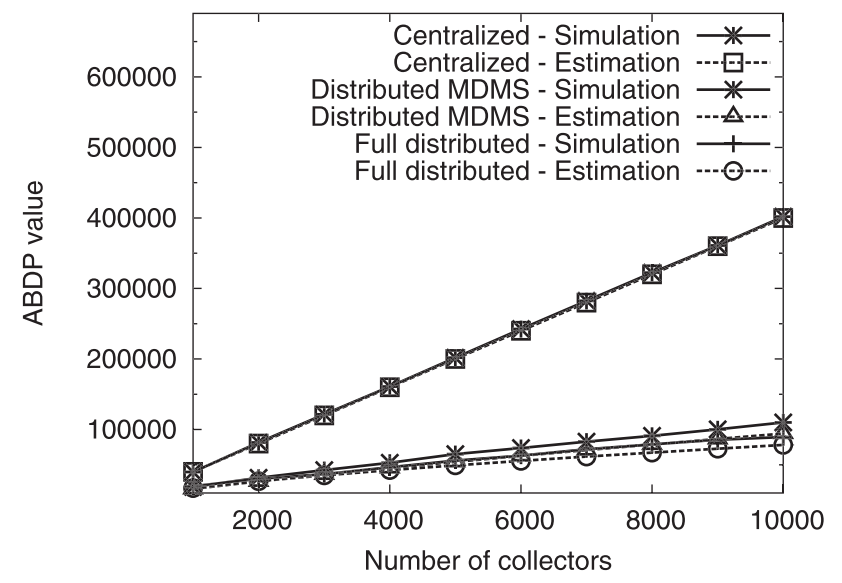

Fig. 5. Comparison of simulation and theoretical estimation results for the ABDP value.

MDMS architecture changes with different parameters. All parameters used will be the same as those in the previous part of this section unless otherwise stated.

In Fig. 6, we present simulation experiments of the effect of the traffic rate $\bar{\lambda}$ on a concentrator. Here, $\bar{\lambda}$ varies from 1 to $10 \mathrm{Mbps}$, which means the sampling interval decreases from 10 seconds to 1 second. It can be observed that in the centralized communication architecture, the total cost of the system scales linearly in terms of $\bar{\lambda}$. In contrast, the total cost in the distributed MDMS communication architecture scales much slower and the difference between its total cost and that of the centralized architecture becomes larger and larger as $\bar{\lambda}$ grows. However, this benefit is less obvious when $\alpha$ becomes large, which means that information usage cost will play a significant role in the total cost. When $\alpha=1$, it actually becomes the same as the centralized architecture since only one MDMS will be selected.

In another simulation experiment, we study the effect of the deployment cost of each MDMS. As demonstrated in Fig. 7, a higher deployment cost will also lead to a higher total cost. Actually, this growing trend can also be observed from our analytical results that are shown in (19): TotCost $\propto \frac{1}{F^{3}}$.

The above observation can also be explained by the internal mechanism of the optimization algorithm. When the

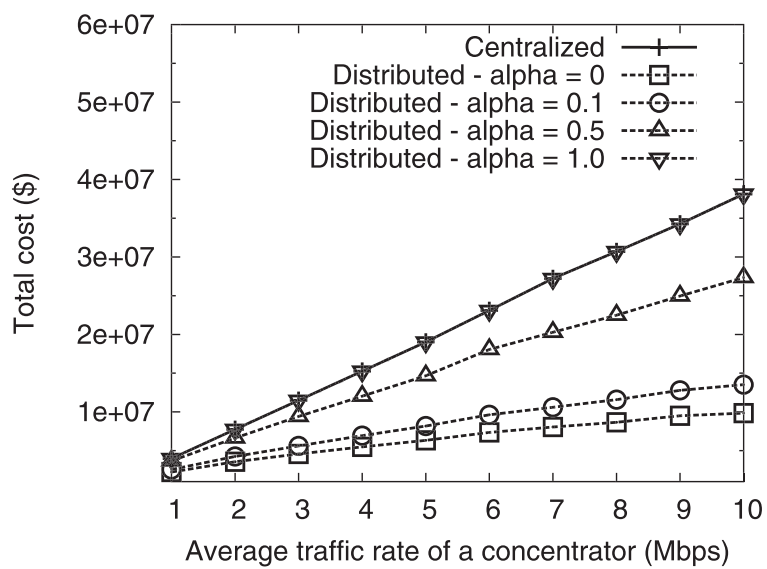

Fig. 6. Change of minimal total cost in terms of average traffic rate on each concentrator.

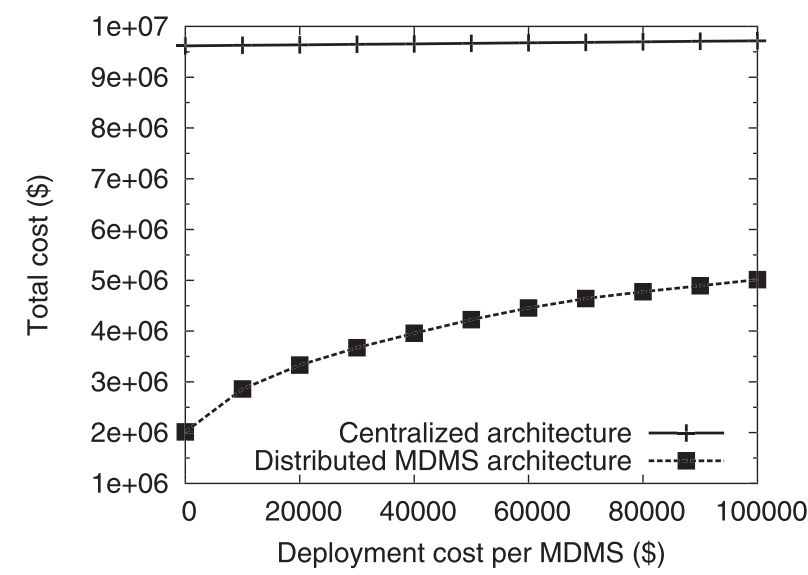

Fig. 7. Minimal total cost in terms of deployment cost of a MDMS.

deployment cost of an MDMS increases, the optimization process will select less number of MDMSs to slow down the increase of the total cost. This is confirmed by the experiment results presented in Fig. 8. In fact, our analysis in (18) shows that $M_{o} \propto \frac{1}{\frac{2}{F^{3}}}$, where $M_{o}$ is the optimal number of MDMS.

If $M$, the number of MDMS candidate locations, is small compared with the theoretical optimal value $M_{o}$, the minimum total cost and ABDP value obtained through Algorithm 1 will be higher than the theoretical optimal results that assume no constraints on selecting locations for MDMSs. As shown in Fig. 9, when $M$ is small, the ABDP value of a distributed MDMS architecture is close to that of the centralized architecture. The minimum ABDP value obtained when there are only four candidate locations is as much as three times of the ABDP value obtained in the situation when there is no limit on the choice of locations.

\section{Conclusion ANd Future Work}

The vast amount of smart meters and the growing needs of highly frequent data readings post a big challenge on the scalability of the smart grid AMI systems. This paper studies two scalable distributed communication architectures that aim to provide efficient smart grid AMI services in facing fast growing traffic from smart meters.

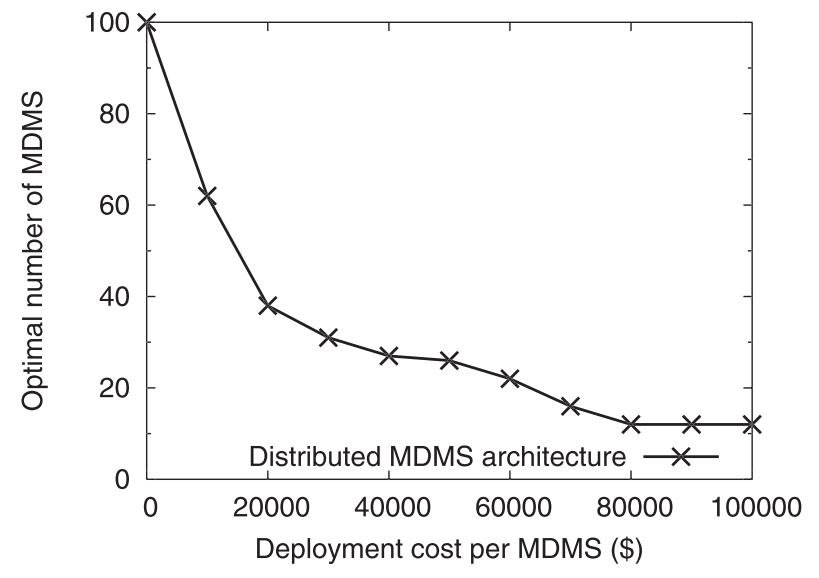

Fig. 8. Optimal number of MDMS versus deployment cost. 


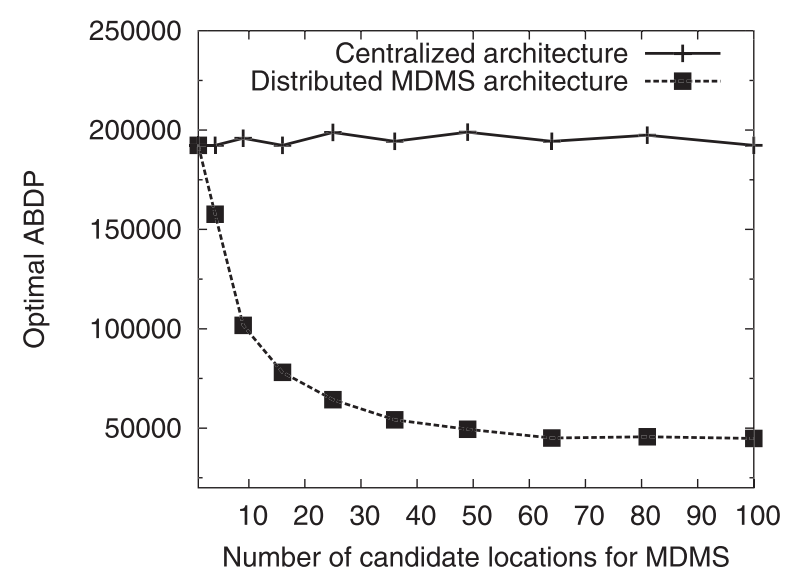

Fig. 9. Optimal ABDP in terms of the number of candidate locations for MDMS.

The placement of MDMSs in a distributed architecture is formulated as an optimization problem to minimize the cost that is proportional to the accumulated bandwidth-distance product, a measurement of communication resources used for data migration in the system to support the smart grid applications. Greedy algorithms are proposed to solve the optimization problems. The simulation results demonstrate that the proposed algorithm can achieve significant benefits in the distributed communication architectures compared with the traditional centralized one.

As another major contribution, an asymptotic analysis is provided to demonstrate the scalability of the distributed communication architectures compared with the traditional centralized communication architecture. The closed-form expressions for the minimal total cost, ABDP value, and the optimal number of MDMSs provide insights into the impacts of different parameters on the communication resources needed.

In the future work, the integration of communication cost scalability and processing ability will be investigated. Furthermore, application of the current work on building a scalable communication architecture into more general cyber-physical systems is a promising new research direction.

\section{REFERENCES}

[1] US Dept. of Energy, National Energy Technology Laboratory, "A Vision for the Modern Grid," 2007.

[2] US Dept. of Energy, Office of Electricity Delivery and Energy Reliability, "Advanced Metering Infrastructure," 2008.

[3] M. Satyanarayanan, "Pervasive Computing: Vision and Challenges," IEEE Personal Comm., vol. 8, no. 4, pp. 10-17, Aug. 2001.

[4] Wikipedia, http://en.wikipedia.org/wiki/Fiber-optic_ communication, 2012.

[5] Wikipedia, http://en.wikipedia.org/wiki/Scalability, 2012.

[6] M. Hill, "What is Scalability?," ACM SIGARCH Computer Architecture News, vol. 18, no. 4, pp. 18-21, Dec. 1990.

[7] P. Jogalekar and M. Woodside, "Evaluating the Scalability of Distributed Systems," IEEE Trans. Parallel And Distributed Systems, vol. 11, no. 6, pp.589-603, June 2000.

[8] http://www.smartgridnews.com/artman/publish/News Blogs_News/The-Coming-Smart-Grid-Data-Surge-1247.html, 2012.

[9] F. Zavoda, "Advanced Distribution Automation (ADA) Applications and Power Quality in Smart Grids," Proc. China Int'l Conf. Electricity Distribution (CICED), 2010.
[10] H. Tram, "Technical and Operation Considerations in Using Smart Metering for Outage Management," Proc. IEEE/PES Transmission and Distribution Conf. and Exposition, 2008.

[11] M. Arenas-Martinez, S. Herrero-Lopez, A. Sanchez, J. Williams, P. Roth, P. Hofmann, A. Zeier, and A., "A Comparative Study of Data Storage and Processing Architectures for the Smart Grid," Proc. IEEE First Int'l Conf. Smart Grid Comm., pp. 285-290, 2010.

[12] S. Rusitschka, K. Eger, and C. Gerdes, "Smart Grid Data Cloud: A Model for Utilizing Cloud Computing in the Smart Grid Domain," Proc. IEEE First Int'l Conf. Smart Grid Comm., pp. 483-488, 2010.

[13] K. Birman, L. Ganesh, and R. Renesse, "Running Smart Grid Control Software on Cloud Computing Architectures," Proc. Workshop Computational Needs for the Next Generation Electric Grid, Apr. 2011.

[14] M. Pathan and R. Buyya, "A Taxonomy and Survey of Content Delivery Networks," Technical Report GRIDS-TR-2007-4, The Univ. of Melbourne, 2007.

[15] E. Lua, J. Crowcroft, M. Pias, R. Sharma, and S. Lim, "A Survey and Comparison of Peer-to-Peer Overlay Network Schemes," IEEE Comm. Surveys and Tutorials, vol. 7, no. 2, pp. 72-93, Apr.June 2005.

[16] I. Stoica, R. Morris, D. Karger, F. Kaashoek, and H. Balakrishnan, "Chord: A Scalable Peer-To-Peer Lookup Service for Internet Applications," Proc. ACM SIGCOMM, 2001.

[17] J. Luo, Q. Zhang, Y. Tang, and S. Yang, "A Trace-Driven Approach to Evaluate the Scalability of P2P-Based Video-onDemand Service," IEEE Trans. Parallel and Distributed Systems, vol. 20, no. 1, pp. 59-70, Jan. 2009.

[18] G. Dan and V. Fodor, "Delay Asymptotics and Scalability for Peerto-Peer Live Streaming," IEEE Trans. Parallel and Distributed Systems, vol. 20, no. 10, pp. 1499-1511, Oct. 2009.

[19] L. Qiu, V.N. Padmanabhan, and G.M. Voelker, "On the Placement of Web Server Replicas," Proc. IEEE INFOCOM, pp. 1587-1596, 2001.

[20] S. Jamin, C. Jin, Y. Jin, D. Raz, Y. Shavitt, and L. Zhang, "On the Placement of Internet Instrumentation," Proc. IEEE INFOCOM, pp. 295-304, 2000.

[21] A. Kuehn and M. Hamburger, "A Heuristic Program for Locating Warehouses," Management Science, vol. 9, no. 4, pp. 643-666, July 1963.

[22] M. Charikar and S. Guha, "Improved Combinatorial Algorithms for Facility Location and k-Median Problems," Proc. IEEE 40th Ann. Symp. Foundations of Computer Science, pp. 378-388, 1999.

[23] C. Gerdes, U. Bartlang, and J. Muller, "Vertical Information Integration for Cross Enterprise Business Processes in the Energy Domain," Proc. Agent-Based Technologies and Applications for Enterprise Interoperability, vol. 25, pp. 1-28, 2009.

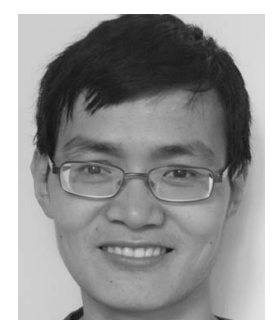

Jiazhen Zhou (S'06, M'09) received the BS degree in mathematics from Shandong University, the MS degree in automatic control from Shenyang Institute of Automation, Chinese Academy of Sciences, and the PhD degree in telecommunication networking from the University of Missouri-Kansas City. Currently, he is working as a postdoctoral researcher with the Department of Computer and Electronics Engineering at the University of Nebraska-Lincoln. His research interests include computer networks, cyber-physical systems, smart grid communications, wireless communications and networks, especially performance analysis and optimal design of communication networks. $\mathrm{He}$ is a member of the IEEE. 


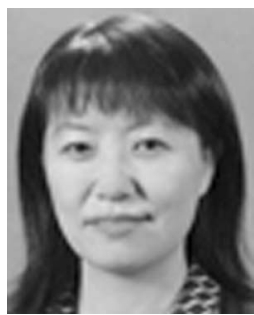

Rose Qingyang Hu (S'95, M'98, SM'06) received the BS degree in electrical engineering from the University of Science and Technology of China, the MS degree in mechanical engineering from the Polytechnic Institute of New York University, and the PhD degree in electrical engineering from the University of Kansas. She has more than 10 years of $R \& D$ experience in the telecommunications industry as a technical manager, a senior wireless system architect, and a senior research scientist. From January 2002 to June 2004, she was an assistant professor with the Department of Electrical and Computer Engineering at Mississippi State University. Currently, she is working as an associate professor with the Department of Electrical and Computer Engineering at Utah State University. She is serving on the editorial boards for Security and Communication Networks, and Wireless Communications and Mobile Computing. She has also been a guest editor for IEEE Communications, IEEE Wireless Communications, and IEEE Network. Her research interests include next-generation wireless communications and networks, network design and optimization, multimedia QoS/QoE, wireless system modeling and performance analysis, and network security. She has published extensively and holds a number of patents in these areas. She is a senior member of the IEEE and a member of Phi Kappa Phi and Epsilon Pi Epsilon Honor Societies.

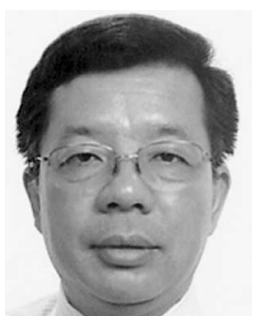

Yi Qian (M'95, SM'07) received the PhD degree in electrical engineering from Clemson University. Currently, he is working as an assistant professor in the Department of Computer and Electronics Engineering, University of NebraskaLincoln (UNL). His research interests include information assurance and network security, network design, network modeling, simulation and performance analysis for next generation wireless networks, wireless ad hoc and sensor networks, vehicular networks, broadband satellite networks, optical networks, high-speed networks and the Internet. Prior to joining UNL, he worked in the telecommunications industry, academia, and the US government. Some of his previous professional positions include serving as a senior member of scientific staff and a technical advisor at Nortel Networks, a senior systems engineer and a technical advisor at several start-up companies, an assistant professor at the University of Puerto Rico at Mayaguez, and a senior researcher at National Institute of Standards and Technology. He has a successful track record to lead research teams and to publish research results in leading scientific journals and conferences. Several of his recent journal articles on wireless network design and wireless network security are among the most accessed papers in the IEEE Digital Library. He is currently serving as the vice chair for Conferences-Communications and Information Security Technical Committee (CISTC) for IEEE Communications Society. He is also serving as the IEEE Communications Society CISTC Representative to the Ad Hoc Committee on Smart-Grid Communications. $\mathrm{He}$ is a member of the ACM and a senior member of the IEEE.

For more information on this or any other computing topic, please visit our Digital Library at www.computer.org/publications/dlib. 